\title{
Align Technology announces UK general dentist forum lineup
}

On 6 December 2020 Align Technology, Inc. (NASDAQ: ALGN), announced that it will stage its annual UK GP Forum on 6 February 2021. The event will be broadcast online from a TV studio. The company has curated a line-up of speakers from both inside and outside of dentistry to explore the most pressing topics shaping the dental sector, which has evolved throughout 2020. The event's programme has been designed to encourage dentists to explore insights into the latest technology and product innovations from Align Technology and beyond.

The speakers include George Blankenship, a former Tesla, Apple and GAP Inc. executive, alongside a notable line-up of dental professionals from the UK and USA. They will be joined by John Morton, Align Director of Research and Technology for product innovation.

Collectively, they will underline the opportunities which exist for general dentists to tap into the growing demand for Invisalign treatment, and embrace the digital advantages of the iTero system. Delegates will learn about the market for new patients in the COVID19 environment, which has changed the dental landscape, and presented some new opportunities.

\section{Keeping ahead of the curve in an evolving market}

The speakers will advocate timely solutions to current issues which may prove challenging even to the most experienced dental professionals. Leading business innovation and transformation advisor George Blankenship will draw on the dynamism of market leading consumer brands to advocate ways to influence positive change amidst the COVID-19 pandemic. Transforming clinical skills, dental practice workflow and patient care will be the domain of the roster of dental professionals on the platform. They will challenge delegates to embrace change, demonstrating that with the right treatments, digital technologies and support, GPs can turn their practices into thriving businesses at the same time as creating new, beautiful smiles for their patients.

A range of clinical presentations aim to inspire delegates to consider the end-to-end patient journey, to evolve their treatment planning and up-skill to treat comprehensively. Finally, R\&D expert John Morton

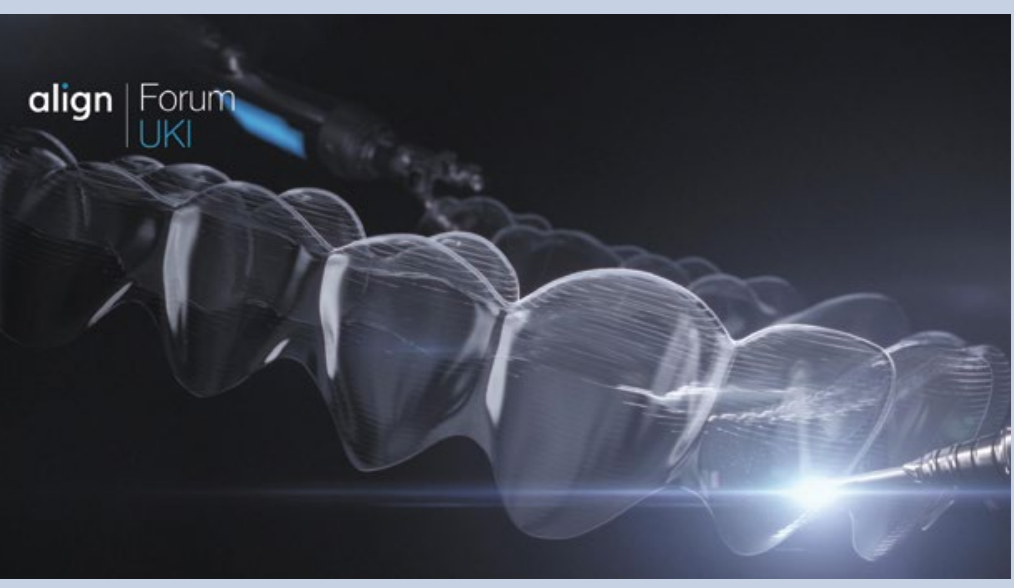

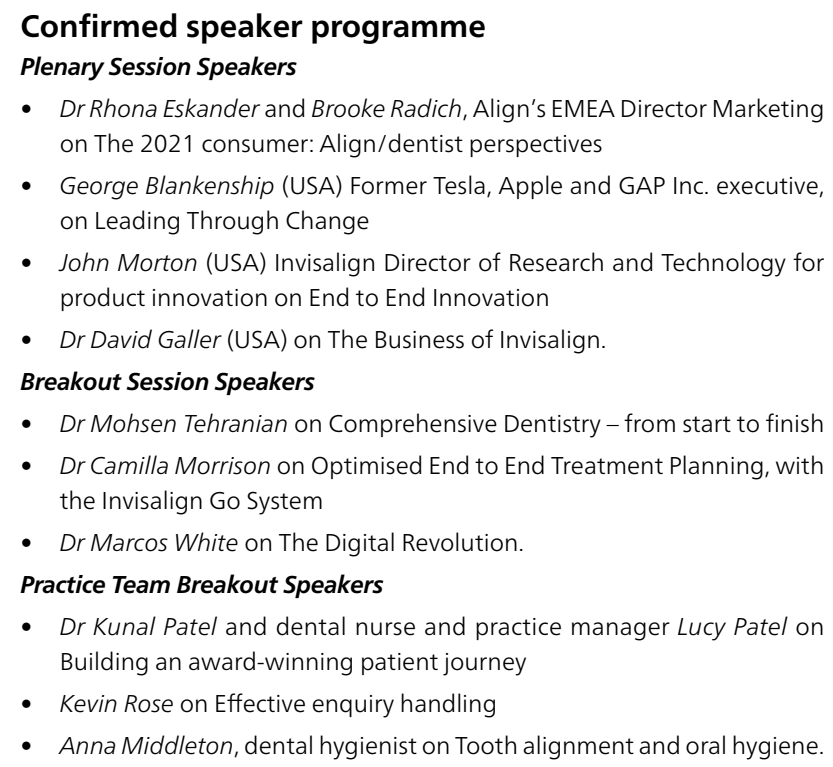

- Dr Rhona Eskander and Brooke Radich, Align's EMEA Director Marketing on The 2021 consumer: Align/dentist perspectives

- George Blankenship (USA) Former Tesla, Apple and GAP Inc. executive, on Leading Through Change

- John Morton (USA) Invisalign Director of Research and Technology for product innovation on End to End Innovation

- Dr David Galler (USA) on The Business of Invisalign.

Breakout Session Speakers

- DrMohsen Tehranian on Comprehensive Dentistry - from start to finish

- Dr Camilla Morrison on Optimised End to End Treatment Planning, with the Invisalign Go System

- DrMarcos White on The Digital Revolution.

Practice Team Breakout Speakers

- Dr Kunal Patel and dental nurse and practice manager Lucy Patel on Building an award-winning patient journey

- Kevin Rose on Effective enquiry handling

- Anna Middleton, dental hygienist on Tooth alignment and oral hygiene.

will describe the collaboration between clinicians and the researchers at Align Technology throughout the last 24 years, illustrating how this has driven innovation and what they can expect next.

\section{Business and clinical growth}

As well as developing the most advanced technologies, the company realised the need to provide practice transformation best practice, education and business support to help practitioners to maximise their practice development. The GP Forum is a piece in the jigsaw of this end-to-end programme of support.

As presenter Dr Marcos White commented: 'The pandemic has just reinforced how important digital technologies have become. I'm delighted to be speaking at the Invisalign UK GP Forum, discussing how the Invisalign system, iTero scanner and digital innovations have helped my business thrive during 2020. I hope that I can instill in my peers that this thriving is better than just surviving'.

Speaker Dr David Galler agrees that digitalisation is a business enabler as it increases patient engagement and helps with treatment uptake: 'The power of visualisation for the consumer is one of the most important things we can do for our patients. In 90 seconds, a patient can visualise how amazing their teeth can look. If my local gym had an outcome simulator and they would show me how I would look after all the gym work, I would probably have the premium gym membership level.

The Invisalign GP Forum will showcase relatable strategies for dental professionals on how to break down barriers to adoption, highlighting the clinical, business and patient centric benefits of digital-centric practice. The topics have been chosen to inspire forward thinking dentists to become dynamic players for 2021 and beyond.

To book a place on the Invisalign UK GP Forum, visit: www. ukgpforum.co.uk 